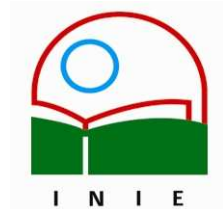

Actualidades Investigativas en Educación

Revista Electrónica publicada por el

Instituto de Investigación en Educación

Universidad de Costa Rica

ISSN 1409-4703

http://revista.inie.ucr.ac.cr

COSTA RICA

\title{
GESTALT Y APRENDIZAJE
}

GESTALT AND LEARNING

Volumen 8, Número 1

pp. 1-12

Este número se publicó el 30 de abril 2008

\section{Fabrizio Fallas Vargas}

La revista está indexada en los directorios:

LATINDEX, REDALYC, IRESIE, CLASE, DIALNET, DOAJ, E-REVIST@S,

La revista está incluida en los sitios:

REDIE, RINACE, OEI, MAESTROTECA, HUASCARAN 


\section{GESTALT Y APRENDIZAJE GESTALT AND LEARNING}

Fabrizio Fallas Vargas ${ }^{1}$

Resumen: El presente trabajo explora las principales implicaciones de la teoría Gestalt en tanto que modelo de aprendizaje. Para ello desarrolla las categorías de análisis fundamental producidas por sus exponentes fundadores, Wertheimer, Koffka, y Köhler, el concepto de pensamiento productivo, así como las reglas perceptuales derivadas de sus investigaciones empíricas. Asimismo se exploran aplicaciones pedagógicas y psicopedagógicas de la teoría, en articulación con el concepto axial de la Gestalt en términos de aprendizaje: die Einsicht, el cual es elaborado en forma crítica.

Palabras clave: GESTALT, APRENDIZAJE POR PERSPICACIA, MODELOS DE APRENDIZAJE, PROCESOS DE APRENDIZAJE, PEDAGOGÍA GESTALTICA, PENSAMIENTO PRODUCTIVO

Abstract: This paper explores the main implications of Gestalt theory as a model of learning. To achieve that purpose, it develops the categories of fundamental analysis conceived by it's founders: Wertheimer, Koffka, and Köhler. It also develops the concept of productive thinking, as well as the rules of perception derived from their empirical investigations. Likewise, it explores pedagogical and psychopedagogical applications of the theory, articulated with the axial concept of Gestalt theory, in terms of learning: die Einsicht, which is elaborated in a critical approach.

Keywords: GESTALT, INSIGHT LEARNING, MODELS OF LEARNING, LEARNING PROCESSES, GESTALTIC PEDAGOGY, PRODUCTIVE THINKING.

Requería Herón II, rey de Siracusa y pariente de Arquímedes (287 a.C. - 212 a.C.), saber si la corona encargada al orfebre local era realmente de oro puro. Advirtió el rey a Arquímedes, expresamente, que no dañase la corona. Arquímedes dio varios rodeos al problema, hasta que un día, al meterse en la bañera, vino a solucionar el asunto. De acuerdo con lo percibido, pensó que el agua que se desbordaba tenía que ser igual al volumen de su cuerpo en ella sumergido, por lo que, trasladando el asunto a la corona de Herón, si medía el agua que rebosaba al meter la corona, accedería a saber el volumen de ésta, y acto seguido, podría compararlo con el volumen de un objeto de oro que pesase igual que la corona. Si los volúmenes no fuesen iguales, sería la prueba de que la corona no era de oro puro. Excitadísimo por el descubrimiento, salió del baño y corriendo desnudo, a palacio, gritaba:

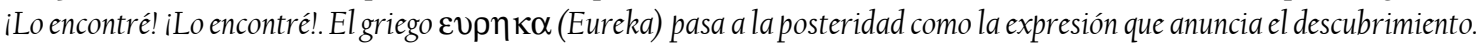
Arquímedes logró demostrar que la corona tenía mayor volumen que un objeto de oro con el mismo peso, contenía plata, un metal de menor densidad que el oro.

"La" fórmula" fundamental de la teoría Gestalt, puede ser expresada de la siguiente forma: Hay todos cuyo comportamiento no está determinado por sus elementos individuales, sino donde los procesos parte se encuentran determinados por la naturaleza intrínseca del todo. Es la esperanza de la teoría Gestalt el determinar la naturaleza de tales todos".

-Max Wertheimer, lección dada en 1924 a la Sociedad Kantiana de Berlín.

\footnotetext{
1 Licenciado en Filosofía de la Universidad de Costa Rica. Profesor de la Escuela de Ciencias Sociales del Instituto Tecnológico de Costa Rica.
}

Dirección electrónica: fv.fabrizius@gmail.com

Artículo recibido: 21 de enero, 2008

Aprobado: 28 de abril, 2008 
El surgimiento de la Gestalt, en tanto que teoría psicológica, completa el panorama de la psicología centroeuropea, junto al estructuralismo, el funcionalismo, que surge hacia finales del siglo XIX y principios del XX, y particularmente, junto al psicoanálisis, por el cual se decantará la Teoría Crítica de la sociedad, el freudomarxismo y las elaboraciones práctico-teóricas de las ciencias sociales progresivas, en abierta oposición política y teóricometodológica contra el positivismo, y, claro está, sus derivas que se constituyen en el campo de la psicología behaviorista, ampliamente extendida al otro lado del Atlántico y la práctica educativa de corte asociacionista, que Max Wertheimer (psicólogo y compositor de origen checo, quien había estudiado leyes en Praga, antes de ir a Berlín a estudiar psicología), procede a criticar en forma vehemente. Con fuerte acento en el idealismo trascendental kantiano, la Gestalt referirá la organización de la percepción en el sujeto a un marco estructurador de lo real a priori, esto es, independientemente de la experiencia.

La noción de Gestalt, es introducida por Christian Von Ehrenfels en 1890, como "forma", "estructura", al descubrir que una misma melodía podría ser tocada sobre distintas notas, al tiempo que las mismas notas en distinto orden daban lugar a una tonada distinta. Siendo que la tonada se da a la percepción, sucede que la totalidad estructural, o sea, la forma, la pone el sujeto. Hacia 1912, Max Wertheimer, realiza experimentos sobre el movimiento estroboscópico (fenómeno phi), junto a los otros grandes exponentes de lo que se denominará en adelante la teoría Gestalt: el berlinés Kurt Koffka, y el estonio, formado en Alemania, Wolfgang Köhler. A partir de tales experimentos, Wertheimer logra concluir que ante la exposición de dos líneas separadas y estacionarias a corta distancia en rápida sucesión temporal, la percepción del sujeto será una línea única moviéndose desde la posición de la primera a la de la segunda línea; movimiento, por lo demás aparente.

Será Köhler quien dará el sentido que tiene entre los psicólogos gestaltistas, el término Gestalt, en tanto que una suerte de entidad característica e individual con existencia independiente y que posee forma, estructura, entre sus atributos.

De acuerdo con Koffka (1935), la aplicación de la Gestalt, significa "determinar que partes de naturaleza pertenecen a todos funcionales, para descubrir su posición en ellos, su grado de independencia relativa y la articulación de grandes todos en sub-todos".

Así las cosas, para la Gestalt, la unidad de análisis fundamental será la noción de estructura, en tanto que organización de los elementos de un todo en sus relaciones, hecha posible por la ley de Praegnanz (pregnancia). Ello es importante puesto que explica el concepto de inteligencia que se maneja en la Gestalt, como la facultad para percibir el campo y la de organizar elementos en orden a la solución creativa (Gestaltungskraft) desde 
lo que propone el conjunto no resuelto (reestructuración) y no la mera acumulación de información dirigida a la reproducción de tareas programadas que propone el modelo de adiestramiento skinneriano. En efecto, como sostiene Wolman (1975, p. 516):

Los chimpancés de Köhler no probaban y erraban al modo de Thorndike, ni se tornaban condicionados al estilo de Pavlov. Abordaban nuevas situaciones; mostraban una orientación en el aprendizaje y bastante inteligencia. No intentaban ciegamente todas las respuestas posibles; su conducta ponía de manifiesto que el aprendizaje incluía un considerable volumen de actividad dirigida a un fin[...]estaban situados en una jaula, y se colocaba un banano a una cierta distancia de ella[...]utilizaban cuerdas, palos y cajas para conseguir el banano; al parecer percibían de algún modo la situación y empleaban la perspicacia para resolver el problema.

El propio Köhler, en la Mentalidad de los simios (1925), registra las siguientes observaciones:

Sultán intenta alcanzar la fruta con el más pequeño de dos palos. Sin éxito, rasga un pedazo de alambre que se proyecta en la red de su jaula, en vano...De repente, recoge de nuevo el palo corto, sube a las barras directamente opuestas a un palo largo, le jala hacia él con el "auxiliar", lo toma, y va con él al punto opuesto en donde se encuentra la meta (la fruta), qué él consigue afianzar.

En ese sentido resulta pertinente la distinción entre pensamiento reproductivo y pensamiento productivo introducida por Wertheimer en Pensamiento productivo:

El pensamiento reproductivo sería aquel que consiste simplemente en aplicar destrezas o conocimientos adquiridos con anterioridad a situaciones nuevas Así, por ejemplo, todos hemos aprendido a aplicar de modo reproductivo la ecuación del 'binomio de Newton' para hallar el cuadrado de una suma: $(a+b)^{2}=\left(a^{2}+b^{2}+2 a b\right)$. En cambio, el pensamiento productivo sería aquel que implicaría el descubrimiento de una nueva organización perceptiva o conceptual con respecto a un problema, una comprensión real del mismo. (Pozo, 1997, p. 171)

Dicha organización de elementos, no por vía generalizadora (como postularía el empirismo anglosajón), sino relacional, tendría como efecto posterior el aprendizaje, cuya metáfora prototípica es el grito arquimédico de Eureka, y ha sido denominada por la Gestalt 
con el nombre de Einsicht, término acuñado por Wolfgang Köhler, con base en sus investigaciones con chimpancés en las islas Canarias. Einsicht remite al aprendizaje por perspicacia, que es un componente clave, al tiempo que polémico de la teoría gestáltica en su conjunto.

De acuerdo con Wertheimer, en Pensamiento productivo, tal como advierte Pozo (1997, p. 172):

...lo fundamental para obtener una solución productiva a un problema y comprenderlo realmente es captar los rasgos estructurales de la situación más allá de los elementos que la componen[...] La solución de problemas y el aprendizaje no se obtendrán por la asociación de elementos próximos entre sí, sino de la comprensión de la estructura global de las situaciones.

La comprensión elemental no reproductiva (propia de simple memorismo asociativo) que se contiene en el Einsicht guarda íntima relación con la ley de Praegnanz, de acuerdo con el cual, en forma a priori, el sujeto gestáltico, tendería a la organización de lo real con base en formas simétricas, completas y regulares, equilibradas y centradas, todo ello en forma inmediata y rápida, tal como lo haría el trascendental kantiano con las intuiciones en las formas puras de la apercepción y las categorías del entendimiento en la Crítica de la razón pura.

La ley de Praegnanz, involucra tres procesos que, de acuerdo a los experimentos gestálticos, intervienen en el aprendizaje: Nivelación o cambio en el sentido de la simetría y la distribución apropiada; agudizamiento, que consiste en la acentuación de los elementos esenciales de una figura, aspecto que le hace fácilmente identificable; normalización que remite a la simplicidad y claridad de la figura percibida. Asimismo, a partir de sus investigaciones, los gestaltistas derivan una serie de reglas de la percepción (que se ilustran en el cuadro 1), y desde luego, el aprendizaje, a saber, la similitud, que remitiría al agrupamiento de datos semejantes en la percepción; la proximidad, en cuyo caso, el agrupamiento de los datos se funda en su carácter próximo; continuidad, por la que son percibidos en términos unitarios los datos agrupados en líneas rectas o curvas; el cierre, cuando se completan los datos que faltan en las figuras que no lo son; fondo-forma, que se operaría en el destaque de figuras contorneadas respecto del contexto que se presenta como fondo, siendo que en caso contrario la confusión de todo el conjunto bloquearía el efecto. 
Los gestaltistas se ocuparon de tareas de aprendizaje de una complejidad mayor a los conductistas, fijados en el ensayo-error y el uso de reforzadores. Así, Wertheimer en Pensamiento Productivo, tal como advierte Pozo (1997, p. 173):

...presenta dos ejemplos de cómo se produce la comprensión súbita de la estructura de los problemas científicos. Analiza el descubrimiento de la teoría de la relatividad por Einstein y la comprensión de la inercia por parte de Galileo. En este último caso, muestra que Galileo descubrió la ley de la inercia cuando se dio cuenta de que el reposo y el movimiento rectilíneo constante eran dos situaciones estructuralmente equivalentes. De esta forma, organizó completamente la estructura conceptual de la mecánica, al interpretar el reposo como un caso de velocidad constante.

Dado que la percepción en sus reglas sería fundamental en tanto que condición productiva del Einsicht, y, en este caso, del aprendizaje, la función del docente es el de identificar el campo perceptivo en el cual este se juega, en términos generales, de organizar los recursos materiales y afectivos para facilitar el alcance de metas que el sujeto que aprende se trazaría dentro de dicho campo. Para ello, Wertheimer distingue tres tipos de procesos que deben ser considerados a nivel pedagógico, a saber: Procesos tipo a que aseguran el pensamiento productivo (que conduce a la relación medios-fines en el contexto de la totalidad) y que, que dan lugar a soluciones decisivas para problemas estructurales. Este tipo de proceso involucra operaciones de agrupamiento, reorganización y el descubrimiento de características esenciales. Por lo demás, no ha de confundirse el Einsicht con procesos de tipo $y$, que se ubican en el discurso pedagógico conductista, el aprendizaje por asociación, el condicionamiento, prueba-error, y que en su confusión, desenfoque y precipitación al extraer conclusiones taran el pensamiento productivo, en el planteo de hipótesis sin sentido y susceptibles de influjo exterior; sino que más bien el Einsicht se verifica en procesos, denominados por Wertheimer, de tipo $b$ que integra la perspicacia y análisis. El pensamiento productivo es el que logra articular relaciones todo-parte y todocualidades en búsqueda de la "verdad estructural", produciendo nuevas y mejores Gestalts,

El aprendizaje, tal como ha sido entendido por Köhler en la Psicología Gestalt, comporta, entonces, una re-estructuración: “...todos los efectos ejercidos por el aprendizaje sobre la subsiguiente experiencia constituyen post-efectos de la organización previa. Si el aprendizaje...equivale a asociación y si es que estamos en lo correcto la asociación es un post-efecto de la organización". (Pozo, 1997, p.175) 
De conformidad con el principio del isomorfismo, el campo perceptivo tendría que ajustarse en su diagnóstico al lenguaje de la física (por lo cual se advertiría una marcada orientación de ciertos elementos de la teoría, sobre todo en el caso de Lewin, por la lectura marburguista del idealismo kantiano). Dicho campo es entonces susceptible de ser diagramado en un esquema topológico espacio-temporalmente delimitado, dentro del cual las percepciones de los sujetos se integrarían, de cara a las metas de aprendizaje propuestas (término que en la Gestalt antagoniza con el elemento de refuerzo conductista) y los impulsos del sujeto, que para estos efectos sería autotélico, en vectores; y en cuyo desplazamiento intervendrían determinadas valencias positivas y negativas cuyo fondo se recorta sobre el carácter de las relaciones que se establecen entre el sujeto percipiente que aprende y sus colegas, así como las que se establecen con el profesor, a nivel particular y grupal. Emerge en este sentido el componente motivacional que el docente ha de tener en cuenta en su accionar dentro de esta relación, así como la propia que apuntala el sujeto en/desde sus vectores, y que sería crucial para la superación de barreras u obstáculos que se presenten dentro del campo para que las metas de aprendizaje sean alcanzadas, en términos de su viabilidad. Lo anterior resulta sumamente interesante, en la medida en que muestra gran afinidad con el planteamiento decrolyano, en tanto que la función globalizadora integra percepción y afectividad, razón por la cual el trabajo mental es susceptible de ser dinamizado por mor de tendencias inmanentes al sujeto y la variabilidad de su estado de ánimo. El fundador de los "centros de interés" va más allá del método de lectoescritura al que comúnmente se reduce su obra, y organiza la ponderación del ambiente de aprendizaje, a nivel curricular, con base en el principio de enseñanza global y una concepción de un sujeto percipiente activo dentro de su entorno:

...la historia (asociación en el tiempo) y la geografía (asociación en el espacio) adquieren una importancia mayor, al igual que las actividades expresivas (lenguaje, dibujo, música, etcétera). Por lo que respecta a la observación Decroly la entiende en la manera más activa posible, casi como exploración del ambiente, y no según el módulo más bien pasivo de las viejas lecciones intuitivas o "lecciones de cosas. (Abbagnano, 1984, p. 669)

La función del docente además de promover el espacio perceptual idóneo (lo cual anuncia el fuerte vínculo de la Gestalt con el enfoque ecológico contemporáneo) debe promover en el estudiante el gestar metas que comprendan la correlación de su todo como sujeto que aprende, a saber, de sus potencialidades, necesidades, limitaciones en su 
momento dado, lo cual conduce a las ventajas del autoplanteamiento de desafíos que le sean provechosos en términos de su formación en tanto que sujeto autónomo. Para alcanzar esta finalidad que Kant identifica con la "salida de la infancia" y la capacidad para pensar por sí mismo, en el contexto revolucionario de la llustración, el docente de la gestalt, adquiere la responsabilidad de proporcionar al sujeto que aprende la confianza en sus propias fuerzas, aspecto de su intervención dentro del campo perceptual que hace sintagma con la selffullfilling prophecy y particularmente con el efecto Rosenthal que plantea el estudio del Pigmalión en sus aplicaciones pedagógicas:

...los alumnos que son valorados positivamente por el docente consiguen mejores resultados que los valorados negativamente, con independencia de sus capacidades reales. Se diría que sobre ellos se proyecta un efecto de confianza en sus propias posibilidades, a la vez que el docente, de forma inconsciente tal vez, actúa con ellos de manera diferente. (Sarramona, 2000, p. 236)

En tal sentido, advierte Sarramona (2000, p. 236), sobre las aplicaciones pedagógicas de la Gestalt, función de estructura y campo perceptual:

La teoría de la Gestalt sugiere la necesidad de plantear las situaciones educativas en general y de aprendizaje en particular teniendo presentes (sic) la situación en su conjunto. El primer contacto con una nueva realidad ha de ser vivida de manera "correcta", esto es, de modo que en el sujeto le quede impregnada una "buena forma". Si las primeras experiencias en una escuela o en el aprendizaje de una materia son negativas, será difícil superar luego esa impresión. Es la aplicación pedagógica del viejo adagio de la vida social que habla de la necesidad de "causar buena impresión desde el primer momento.

En virtud de lo anterior, la Gestalt, demanda una organización del campo perceptual de aprendizaje dentro de una totalidad no inserta sino fluyente en sus elementos (cuya dinámica se encuentra en la del todo). En forma coherente el planteamiento de las actividades, procesos en los que se involucra el sujeto gestáltico que se (auto)organiza en el aprendizaje, demanda del docente, además, el debido cuidado en torno a la experiencia primera, en la que el sujeto se interrelaciona con el objeto de la percepción, su adecuada presentación remite a la necesaria consideración de que el puro objeto no resulta, pese a ser inmediatamente organizable, sino que dicho objeto ha de representar para el sujeto una "buena forma", esto es, que el momento de planeamiento resulta fundamental, así como la 
flexibilidad del proceso de aprendizaje en marcha. El objeto no puede por sí mismo indicar nada si no es para el sujeto que ha de otorgarle sentido, forma, y es en este momento en donde la Gestaltungskraft (la fuerza creativa) requiere del docente la percepción de estructura, en la que el cierre se haya comprendido, y el inicio ha de contener la buena forma en consecuencia, para que el sujeto gestáltico que aprende progrese en el uso de sus fuerzas creativas, y proceda a la producción de lo no manifiesto (lo contrario sería reducir la función de estructura a suma de elementos atomísticos) en lugar de la mera reproducción.

De acuerdo con Wertheimer es deseable el desarrollo de métodos para la descripción y medida de cualidades totales. La diferenciación entre enfoque y reenfoque es fundamental para comprender los contornos del pensamiento productivo, tal como apunta Wolman (1975, p. 520):

En el enfoque, se produce un cambio o transición desde un punto de vista subjetivo o personal a un punto de vista más independiente con una aprehensión objetiva de la situación total y de los requisitos estructurales y funcionales. Esta operación da lugar al dominio de los requisitos estructurales y funcionales objetivos de la situación y a una neutralización de la interferencia de las propias creencias y experiencias personales. El reenfoque consiste en la obtención de una perspectiva nueva y penetrante. Proporciona un nuevo ángulo desde el cual considerar la cuestión de los logros e intereses múltiples de las personas creativas, y la capacidad de los científicos e ingenieros, adiestrados en determinados terrenos para funcionar, tras un breve intervalo de orientación, en otros terrenos con altos niveles de productividad.

La función psicopedagógica a nivel de planeamiento, asesoramiento e intervención es fundamental dentro de este campo perceptivo de aprendizaje gestáltico, siendo que los vectores en razón de los cuales el sujeto se encuentra topológicamente dirigido al alcance de determinado objetivo, por ejemplo, u objetivo de aprendizaje, entran en relación con elementos que a su vez resultan determinantes en el contenido específico de las valencias en que se juega el carácter significativo del campo perceptual de aprendizaje (Ver Figura 1). Dichos elementos integran necesidades, que en el caso del sujeto que aprende son particulares, puesto que si bien la Gestalt postula el carácter a priori de las reglas de organización perceptual, admite su especificidad interpretativa y personalísima (ya Köhler en sus estudios sobre la naturaleza de las asociaciones, mostraba la falsedad de principio asociacionista de equipotencialidad, según el cual todas las asociaciones se aprenden con la misma facilidad); de ahí que tales necesidades puedan estar vinculadas a estilos, ritmos y/o 
necesidades especiales de aprendizaje, y cuyo abordaje psicopedagógico resulta crucial de acuerdo con el principio de atención a la diversidad, al ingresar dentro del campo valorativo no solamente las áreas por mejorar (componentes extraídos del proceso diagnóstico), sino además, en equilibrio con las potencialidades que caracterizan al sujeto, esto es, sus habilidades perceptuales, herramientas (meta)cognitivas, recursos psicofísicos, afectivos y las estrategias que utiliza para producir soluciones (no simplemente a resolver tareas o problemas en forma eficiente) que le permitan hic et nunc, alcanzar los objetivos propuestos dentro del espacio vital de aprendizaje, habida cuenta de posibles barreras que se fraguan dentro del ambiente perceptual y psicológico y que se expresan como valencias positivas y negativas que estarían siendo provechosas o perniciosas para la organización del aprendizaje. Ello es fundamental para comprender cómo mediar procesos de aprendizaje en forma efectiva dentro del espacio-vital perceptual de aprendizaje que es el aula (una de las derivas contemporáneas de influencia gestáltica es el denominado enfoque ecológico), en términos de la gestalt, y desde luego para comprender la necesaria coordinación de los elementos que componen la dinámica de un sistema-forma con mayor capacidad de absorción, como habría de serlo en su expresión a nivel institucional, esto es a los elementos involucrados directamente en el proceso de aprendizaje (docentes-discentes), y otros cuyo involucramiento es más remoto (autoridades institucionales) y/o peculiar: ámbito familiar y comunidad; además las necesidades que presentan los sujetos que integran tales ámbitos, la clarificación de las fortalezas y elementos por mejorar, a nivel curricular, de infraestructura, clima organizacional que envuelve a su vez el conjunto de espacios perceptuales y de aprendizaje en un momento dado dentro de los contornos físicos de determinada institución al interior del subsistema educativo, que a su vez se plantea fines formativos y comprende una serie de áreas fuertes, necesidades, limitaciones, barreras que remiten al propio carácter de la formación económico social a la que sustenta y reproduce como aparato ideológico. 
FIGURA 1. DIAGRAMA TOPOLOGICO DEL CAMPO PERCEPTUAL DE APRENDIZAJE

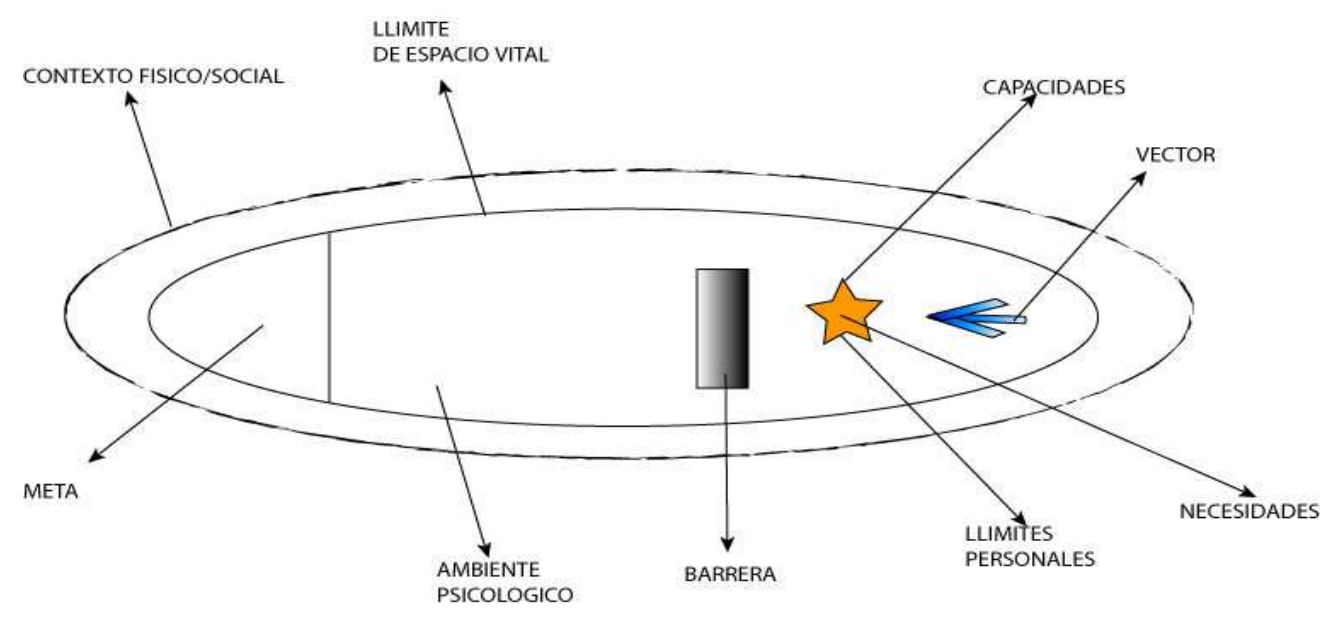

Uno de los aspectos polémicos que remiten no sólo al sujeto percipiente sino al subsistema educativo, siendo clara su función en el mantenimiento del statu quo, y que la Gestalt recupera con gran fuerza crítica es el problema del traído y llevado "conocimiento previo" o la "experiencia previa" que no necesariamente podría entenderse como experiencia, puesto que ésta última tendería a la reestructuración, como sabía Vigotsky, apertrechado de la dialéctica hegeliana y marxista, y no a la mera estructuración típica. La polémica que carga el concepto de Einsicht se encuentra imbricada en su tendencia homologadora del campo perceptual y el de aprendizaje no asimilatorio o reproductivo, por lo que no alcanza a explicar la producción del Einsicht, dibuja ciertamente sus contornos, pero el enfoque idealista siempre es apriorístico, y su sujeto prehegeliano carece de historia, por ello la insistencia hic et nunc de la percepción y del aprendizaje. No obstante el momento de verdad del planteamiento gestáltico es el que genera este conflicto en que se resuelve su propia contradicción:

...La Gestalt no proporciona una explicación sobre la influencia de la naturaleza pasada en la comprensión súbita de un problema. De hecho, el efecto de la experiencia previa más estudiado por los gestaltistas, la fijeza funcional, está relacionado más bien con la influencia negativa de esa experiencia, que, según sus investigaciones, en ciertas circunstancias vendría a dificultar la reestructuración del problema en lugar de facilitarla [...]cuando una tarea o problema tenga varias estructuras posibles y alguna de ellas resulte más inmediata o fácil de percibir para el sujeto, la reestructuración resultará más difícil. Igualmente cuando en la solución de una tarea entren en juego intereses o motivos personales, el cambio a una estructura 
distinta de la situación se verá obstaculizado (Wertheimer, 1945). En ambos casos la fijeza funcional o resistencia a reestructurar la tarea impedirán su correcta solución y, por tanto, el aprendizaje productivo. (Pozo, 1997, p.175)

Los casos estudiados por Wertheimer, de la historia de la ciencia, Einstein y Galileo, requieren incorporar un lapso de preparación o incubación, pero ello liga el Einsicht a sus contornos, sin solución de continuidad entre percepción, con todas sus determinaciones psicofísicas y conocimiento, tal es la función del concepto de meta en la gestalt, que vendría a resemantizar el valor de la tarea inconclusa, en su transición justamente al alcance de esa meta. Abstraída la fijeza funcional de la experiencia previa, el concepto de aprendizaje por reestructuración (sin consideración dialéctico-materialista) tiene que retrotraer a los rasgos definitorios del sujeto trascendental kantiano, quien con base en sus categorías a priori organiza lo real y así le produce. Nótese que el concepto de fijeza funcional, al involucrar el uso no meramente teórico de la razón, sino práctico-empírico, no deja de mostrar la aversión kantiana por el tema de lo patológico, esto es, la implicación de las inclinaciones que vendrían a entorpecer, a fraguar ilusiones que pertenecen al sujeto empírico, contra la cientificidad y rigor del sujeto en su uso teórico de la razón crítica. Algo de la mediación tendría que abstraerse para salvar a la inmediatez de sí misma, y en ello la Gestalt apunta bien, de ahí que sea éste, su momento emancipatorio, aunque debe entenderse dentro de la lógica del sujeto trascendental, por ello no puede resolver, cuando la resolución del problema es de carácter extralógico. El modelo local, formalmente constructivista, habría de incorporar dicho momento emancipatorio, posicionando la función material de la educación allende la realidad que cumple y clausura parodia del modelo conductista, cuya corporeización se efectualiza en la estructuración académica institucional del docente transmisor de "cultura" no ceja en sus esfuerzos de realizar, al proyectarse desde ellos, currículum oculto: el Einsicht de la dominación.

\section{Referencias}

Abbagnano, Niccola. (1984). Historia de la pedagogía. México: Fondo de Cultura Económica.

Koffka, Kurt. (1935). Principles of Gestalt Psychology. Recuperado el 12 de noviembre del 2007 de http://www.marxists.org/reference/subject/philosophy/works/ge/koffka.htm

Köhler, Wolfgang. (1925). La mentalidad de los simios. Recuperado el 12 de noviembre del 2007 de http://www.kirjasto.sci.fi/kohler.htm. 
Pozo, Juan. (1997). Teorías cognitivas del aprendizaje. Madrid: Morata.

Sarramona, José. (2000). Teoría de la Educación. Reflexión y normativa pedagógica. Barcelona: Ariel.

Wertheimer, Max. (1924). Gestalt theory. Recuperado el 12 de noviembre del 2007 de http://www.gestalttheory.net/archive/wert1.html\#fn1.

Wolman, Benjamín. (1975). Teorías y sistemas contemporáneos de psicología. Barcelona: Martínez Roca. 